Cahiers
de a $\begin{gathered}\text { Recherche } \\ \text { sur les Droits } \\ \text { Fondamentaux }\end{gathered}$

Cahiers de la recherche sur les droits fondamentaux

$12 \mid 2014$

Droit et psychiatrie

\title{
La responsabilité administrative de l'établissement pratiquant du soin psychiatrique
}

Éric Péchillon

\section{OpenEdition}

Édition électronique

URL : http://journals.openedition.org/crdf/1916

DOI : $10.4000 /$ crdf.1916

ISSN : 2264-1246

Éditeur

Presses universitaires de Caen

Édition imprimée

Date de publication : 1 décembre 2014

Pagination : 73-85

ISBN : 978-2-84133-507-7

ISSN : 1634-8842

Référence électronique

Éric Péchillon, «La responsabilité administrative de l'établissement pratiquant du soin psychiatrique », Cahiers de la recherche sur les droits fondamentaux [En ligne], 12 | 2014, mis en ligne le 01 octobre 2015, consulté le 10 décembre 2020. URL : http://journals.openedition.org/crdf/1916 ; DOI : https://doi.org/ $10.4000 /$ crdf. 1916

Cahiers de la recherche sur les droits fondamentaux 


\title{
La responsabilité administrative de l'établissement pratiquant du soin psychiatrique
}

\author{
Éric PÉCHILLON \\ Maître de conférences en droit public à l'université de Rennes 1 \\ Institut du droit public et de la science politique (IDPSP)
}

I. La mauvaise prise en charge du patient

A. Le patient en soins libres: I'application du droit commun de la responsabilité hospitalière et la place exceptionnelle du recours à la contrainte

B. Le patient sous contrainte: l'absence de présomption de faute au profit d'une analyse circonstanciée de chaque situation

II. Les dommages causés par les patients admis en psychiatrie

A. Les dommages causés par un patient en soins libres

B. Les dommages causés par un (ex-)patient en soins sous contrainte

III. L'atteinte aux droits fondamentaux de la personne accueillie dans un service de psychiatrie

A. Repenser les règles de fonctionnement des services de psychiatrie afin de respecter les personnes prises en charge

B. Informer la personne dans un langage accessible

La réforme du soin psychiatrique sous contrainte a été l'occasion de s'interroger sur l'étendue des missions et des prérogatives confiées aux établissements chargés d'accueillir des patients souffrant de troubles mentaux. En combinant des dispositions relatives à la police administrative et au service public, le législateur a confié à certains établissements de santé une mission particulièrement délicate à mettre en œuvre puisqu'il s'agit de trouver le juste équilibre entre des objectifs a priori antinomiques. Accueillir des personnes contre leur gré n'est jamais chose aisée. Lorsque celles-ci souffrent de pathologie mentale, la tâche est encore plus délicate, tant les situations individuelles sont variées. La vulnérabilité des patients, le caractère imprévisible de certains comportements, la soudaineté de certains passages à l'acte sont autant d'éléments à prendre en considération dans l'organisation des services et la formation des personnels. Dès l'instant où la surveillance des patients est confiée à un établissement, il n'est pas vraiment surprenant que la question de l'engagement de la responsabilité administrative des établissements finisse par se poser ${ }^{1}$.

1. À noter que bien avant l'unification du contentieux devant le juge des libertés et de la détention, le Tribunal des conflits considérait qu'il revenait au juge judiciaire de statuer sur la réparation du préjudice subi du fait d'un arrêté de placement d'office, y compris lorsque l'illégalité de l'arrêté résultait d'un défaut de procédure (TC, Préfet de la région Île-de-France, 17 février 1997, n 97-03045, Recueil Lebon, p. 525; La semaine juridique, éd. G, 1997, II, 22885, concl. J. Sainte-Rose; Droit administratif, nº 138, 1997, note M. Paillet; CA Paris, 31 mai 1996, Dalloz, 1996, IR 165 et 1997, p. 554, note J. Prévault; La semaine juridique, éd. G, 1997, II, 22955, note G. Fauré) : «Considérant que si l'autorité judiciaire est seule compétente, en vertu des articles L. 333 et suivants du Code de la santé publique, pour apprécier la nécessité d'une mesure de placement d'office en hôpital psychiatrique et les conséquences qui peuvent en résulter, il appartient à la juridiction administrative d'apprécier la régularité de la décision administrative qui ordonne le placement; que lorsque cette dernière s'est prononcée sur ce point, l'autorité judiciaire est compétente pour statuer sur les conséquences dommageables de l'ensemble des irrégularités entachant la mesure de placement d'office» (TC, Préfet de la région Île-de-France) 
Si la psychiatrie ne se résume fort heureusement pas à la contrainte, cette dernière est souvent le biais par lequel le juge est amené à examiner le fonctionnement des établissements de santé mentale. Souvent les affaires mettant en cause leur responsabilité obligent le juge à s'interroger sur la manière dont l'institution fait usage des prérogatives de puissance publique que lui a accordées le législateur. Un emploi disproportionné de la contrainte mais également une levée trop rapide de celle-ci sont souvent le point de départ d'un recours contentieux visant à obtenir l'indemnisation des préjudices subis. À partir de quelques affaires récentes, il est possible de dresser un rapide panorama des questions posées par ce type de contentieux. Il ne s'agira pas ici de faire une présentation exhaustive du droit de la responsabilité administrative mais plutôt de saisir les mutations auxquelles est confronté l'hôpital psychiatrique depuis la réforme de 2011. Il est possible de regrouper les différentes affaires autour de trois grandes thématiques: la mauvaise prise en charge du patient (I); les dommages causés par les patients admis en psychiatrie (II); l'atteinte aux droits fondamentaux de la personne accueillie dans un service de psychiatrie (III).

\section{La mauvaise prise en charge du patient}

Dans ce type d'affaire, la victime principale est le patient (ou son ayant droit pour les dommages les plus graves) qui considère que les soins apportés par l'établissement n'ont pas permis d'éviter la survenance d'un préjudice. La mort accidentelle ou le suicide d'un patient souffrant de troubles psychiatriques est ainsi l'occasion de s'interroger sur l'existence d'une faute susceptible d'engager la responsabilité de l'établissement de soins. Le passage à l'acte suicidaire est l'une des causes de prise en charge dans les services de psychiatrie. Le patient peut alors être pris en charge à sa demande en soins libres, mais il peut aussi faire l'objet d'un placement en soins à la demande d'un tiers ${ }^{2}$, voire parfois d'un placement en soins psychiatriques à la demande du représentant de l'État (SDRE). L'usage de son corps est souvent considéré comme une liberté individuelle. Il arrive cependant que dans certaines situations le législateur habilite les soignants à user de la contrainte afin d'éviter des comportements qui peuvent conduire à la mort de la personne. À ce titre, le geste suicidaire peut parfois être considéré comme lié à la pathologie mentale. Même si, chaque année, près de 200000 personnes tentent de mettre fin à leurs jours, ce n'est que sous certaines conditions très précises que la responsabilité de la puissance publique peut être retenue. La lutte contre le suicide fait l'objet d'un plan national 2011-2014 mobilisant les services de six ministères, avec une coordination territoriale confiée aux agences régionales de santé (ARS). Il n'est alors pas aisé de trouver le juste équilibre entre la préservation de la liberté individuelle et la nécessaire prise en charge et surveillance du patient. Les politiques de santé publique n'ont pas vocation à socialiser l'ensemble des risques ni à élargir le champ de la responsabilité sans faute.

\section{A. Le patient en soins libres: l'application du droit commun de la responsabilité hospitalière et la place exceptionnelle du recours à la contrainte}

Chaque affaire portant sur la responsabilité d'un établissement va obliger à qualifier précisément la situation juridique dans laquelle se trouvait la victime. Il est important de rappeler dès à présent que le patient en soins libres n'est pas dans une situation identique à celle d'un patient faisant l'objet d'une mesure de soins sous contrainte, et qu'une personne retenue contre son gré dans un établissement de santé sans faire l'objet d'une hospitalisation sans consentement serait victime d'une voie de fait ${ }^{3}$ dès lors qu'elle serait retenue au-delà du temps strictement nécessaire pour transformer la prise en charge.

Lorsqu'un patient est en soins libres, il n'est pas possible à l'équipe soignante d'avoir recours à la contrainte en dehors des situations d'urgence. Les obligations à la charge du service ne sont d'ailleurs pas exactement les mêmes. Parmi les nombreuses missions confiées aux équipes soignantes, la prévention de l'acte auto-agressif, voire suicidaire, est une préoccupation constante. Il n'existe cependant pas sur cette question d'obligation de résultat à la charge du service public hospitalier. Si cela était le cas, le risque serait d'aboutir à un régime d'hospitalisation basé sur la présomption de dangerosité, sur l'interdit et la suspicion généralisée. Depuis la loi du 27 juin 1990,

2. Soins à la demande d'un tiers (SDT); soins à la demande d'un tiers, en cas d'urgence (SDTU); soins en cas de péril imminent (PI).

3. CE, $M^{m e}$ Brousse, 18 octobre 1989, n 75096 , mentionné aux tables du Recueil Lebon, p. 938; Dalloz, 1989, IR 309; La semaine juridique, éd. G, 1989, IV, 415; L'actualité juridique. Droit administratif, $\mathrm{n}^{\circ}$ 1, 1990, p. 56, concl. B. Stirn: «Considérant qu'une personne majeure présentant des signes de maladie mentale, ne peut être retenue contre son gré dans un établissement d'hospitalisation que pendant le temps strictement nécessaire à la mise en œuvre des mesures d'internement d'office ou de placement volontaire, prévues par le code de la santé publique; que la famille de $\mathrm{M}^{\mathrm{me}} \mathrm{B}$. s'étant refusée à demander son placement volontaire, il appartenait à l'administration hospitalière, dans le cas où les médecins de l'hôpital estimaient que le maintien de la patiente en milieu psychiatrique s'imposait, dans son intérêt ou celui des tiers, de demander à l'autorité préfectorale d'user des pouvoirs qu'elle tient des dispositions du code de la santé publique, et, notamment, de son article L. 350 ; que, dès lors, en l'absence de tout titre l'autorisant légalement, le maintien contre son gré de $\mathrm{M}^{\mathrm{me}} \mathrm{B}$. dans le service psychiatrique de l'hôpital Lariboisière a constitué une voie de fait;/Considérant que l'action en réparation de l'ensemble des dommages résultant de cette voie de fait relève de la compétence des tribunaux judiciaires; qu'il en va ainsi tant du préjudice résultant de la privation de liberté du fait du maintien contre son gré de $\mathrm{M}^{\mathrm{me}} \mathrm{B}$. en service psychiatrique, que des dommages qui résulteraient du traitement qui lui a été administré durant son hospitalisation et des informations qui, au cours de cette même période, auraient été données par l'hôpital à son employeur sur la nature de l'affection diagnostiquée; que, dès lors, il y a lieu d'annuler le jugement en date du 4 décembre 1985 par lequel le tribunal administratif de Paris s'est reconnu compétent pour connaître de la demande de $\mathrm{M}^{\mathrm{me}} \mathrm{B}$. et de rejeter sa demande de première instance comme portée devant une juridiction incompétente pour en connaître». 
l'hospitalisation libre constitue le mode privilégié de prise en charge du patient psychiatrique vers lequel il faut tendre dès que possible. À l'inverse, le soin sous contrainte doit être conçu comme une exception strictement encadrée par le législateur. Le Parlement a d'ailleurs bien pris soin de rappeler en 2011 qu' «une personne ne peut sans son consentement ou, le cas échéant, sans celui de son représentant légal, faire l'objet de soins psychiatriques ${ }^{4}$. La contrainte doit par conséquent être exceptionnelle et être utilisée dans le cadre fixé par le législateur 5 .

Ce principe entraîne des conséquences importantes à la fois sur les droits des patients et sur les obligations à la charge du service. À titre d'illustration, $\mathrm{M}^{\mathrm{me}} \mathrm{C}$. était accueillie dans un service psychiatrique sous le régime de l'hospitalisation libre ${ }^{6}$. Vingt-six jours plus tard, comme elle en avait le droit puisqu'elle ne faisait pas l'objet d'une hospitalisation sous contrainte, elle a quitté l'établissement. Très peu de temps après, elle s'est précipitée dans le vide du haut d'un viaduc urbain. Devenue paraplégique, elle souhaite voir engagée la responsabilité de l'hôpital qui, selon elle, aurait dû l'empêcher de quitter l'établissement. La Caisse primaire d'assurance maladie (CPAM) du Puy-de-Dôme se joint à l'instance en vue d'obtenir le remboursement des frais de dépenses de santé présents et futurs. En appel, les juges ${ }^{7}$ avaient refusé de reconnaître l'existence d'une faute commise par l'institution hospitalière en estimant que la liberté de circulation des individus devait demeurer la règle tant que les circonstances n'imposaient de modifier la prise en charge. Le Conseil d'État confirme cette analyse en précisant l'importance du mode de prise en charge sur l'application du régime de responsabilité. Les obligations à la charge de l'institution hospitalière ne sont en effet pas les mêmes pour l'hospitalisation libre qu'en matière de soins sous contrainte. Dans le premier cas, lorsqu'un patient quitte l'établissement, il s'agit juridiquement d'une sortie contre (ou sans avis) avis médical et dans le second d'une «fugue». Dans les deux situations, les services soignants se doivent de réagir mais en tenant compte des conditions juridiques de la prise en charge. Il n'est en effet pas question d'user de la force et de la coercition contre un patient admis en hospitalisation libre. Les seuls moyens d'action offerts à l'équipe soignante, lorsqu'elle estime que la sortie de l'établissement et l'arrêt des soins sont problématiques, consistent à tenter de «faire entendre raison au patient» ou d'alerter l'autorité administrative compétente afin que celle-ci modifie dans les plus brefs délais la prise en charge (PI ou SDRE).

Dans cette affaire, personne ne contestait la réalité des troubles psychiatriques dont souffre la victime. L'expert désigné en référé par le président du tribunal administratif rappelle que la patiente avait été hospitalisée à plusieurs reprises à partir de 1994 et qu'elle présentait, en avril 2006, «une pathologie psychotique du registre de la schizophrénie avec extériorisation polymorphe qui a conduit à la prescription de traitements psychotropes et de mesures de surveillance». La discussion portait surtout sur l'existence de fautes qui auraient été commises par le personnel soignant et l'hôpital. Fallait-il ne pas respecter le choix d'une patiente? Pour écarter définitivement la responsabilité de l'établissement, le Conseil d'État insiste sur l'importance du mode d'hospitalisation. Lorsqu'un patient entre dans un service librement et consent aux soins, il «dispose des mêmes droits liés à l'exercice des libertés individuelles que ceux qui sont reconnus aux malades hospitalisés pour une autre cause ${ }^{8}$. Ce renvoi à l'esprit du Code de la santé publique est primordial à la fois pour les patients et pour les soignants. D'un côté, il permet de ne pas stigmatiser la maladie mentale et de respecter les droits fondamentaux de la personne humaine, y compris lorsqu'elle souffre de pathologie mentale. De l'autre, il offre à l'établissement de santé un cadre d'action plus prévisible. À chaque type de prise en charge correspondent une réglementation et des obligations particulières. Il n'est pas possible pour l'équipe soignante d'en modifier unilatéralement le contenu en basculant vers un régime de soins sous contrainte. Elle est uniquement tenue de proposer des traitements adaptés à la pathologie et à son évolution. Si l'état du patient suppose l'instauration d'un régime contraignant, les soignants ne peuvent que signaler cette dégradation aux personnes compétentes pour enclencher une telle procédure (représentant de l'État, directeur d'établissement ou tiers). Ayant sollicité sans succès de la part de l'entourage du patient une transformation en hospitalisation à la demande d'un tiers (ce qui correspondrait désormais à une admission sur décision du directeur d'établissement à la demande d'un tiers), le service n'a commis aucune faute puisque l'état du patient ne permettait pas d'envisager une mesure préfectorale (ADRE: admission sur décision du représentant de l'État). Le juge administratif s'appuie enfin sur l'avis de l'expert qui rappelle l'imprévisibilité du passage à l'acte : la «prise en charge était [...] adaptée à sa pathologie, laquelle, selon ses conclusions, ne manifestait aucun élément dépressif ou mélancolique et ne pouvait laisser présager de quelconques idées suicidaires ». En procédant de la sorte, le juge évite l'écueil d'une analyse globale de la prise en charge de la maladie mentale. Il refuse toute idée de socialisation du risque de passage à l'acte et replace la faute de service au cour du débat contentieux. Cela ne signifie nullement qu'il soit impossible d'obtenir une indemnisation des préjudices subis lors d'un traitement psychiatrique. Il faudra simplement parvenir à démontrer l'existence d'une faute dans l'organisation ou le fonctionnement du service. 
On pourra par exemple reprocher à l'établissement de ne pas s'être assuré qu'un patient continuait bien à suivre son traitement. La cour administrative de Lyon ${ }^{9}$ adopte d'ailleurs une solution en ce sens lorsqu'elle estime responsable l'établissement de santé qui, à la suite d'une sortie contre avis médical d'un patient souffrant de schizophrénie paranoïde, n'a pris aucune mesure pour s'enquérir de la manière dont le patient évoluait. Il est donc conseillé aux équipes soignantes d'évaluer la situation d'un patient en rupture de traitement, en prenant en considération la nature de la pathologie. Sans pour autant lever le secret médical, il conviendra donc à l'établissement de prendre toutes les mesures utiles visant à prévenir tout nouveau passage à l'acte.

\section{B. Le patient sous contrainte: I'absence de présomption de faute au profit d'une analyse circonstanciée de chaque situation}

La situation n'est pas exactement identique lorsque le patient fait l'objet d'une prise en charge sous contrainte, en particulier suite à la demande d'un tiers. En effet, dans ce cas, le patient est considéré comme une personne vulnérable qu'il convient de protéger d'elle-même. La loi fixe deux conditions cumulatives permettant d'user de la contrainte à son encontre:

Une personne atteinte de troubles mentaux ne peut faire l'objet de soins psychiatriques sur la décision du directeur d'un établissement [...] que lorsque les deux conditions suivantes sont réunies: $1^{\circ}$ Ses troubles mentaux rendent impossible son consentement; $2^{\circ}$ Son état mental impose des soins immédiats assortis soit d'une surveillance médicale constante justifiant une hospitalisation complète, soit d'une surveillance médicale régulière ${ }^{10}$.

L'absence de consentement est la pierre angulaire de ce dispositif puisqu'elle permet d'user de prérogatives de puissance publique dans l'intérêt de la personne (et donc indirectement dans l'intérêt général). Cependant, les différentes juridictions qui ont eu à examiner les modalités pratiques de prise en charge de ces patients vulnérables refusent de considérer que l'ensemble des personnes accueillies forme une masse homogène. Au contraire, chaque situation est examinée individuellement en fonction du profil et de l'évolution de chaque patient. Il est ainsi possible de rechercher s'il existe une forme d'adhésion d'un patient pourtant juridiquement incapable de consentir. En psychiatrie, la distinction entre l'adhésion aux soins et le consentement est primordiale, en particulier pour l'instauration d'un programme de soins. Pour le juriste, l'absence de consentement ne signifie pas la perte totale de liberté et la disparition du libre arbitre. Un patient incapable de consentir peut parfaitement rester responsable de ses actes.

Dans une société où la mort prématurée est souvent perçue comme un échec collectif, la question du suicide occupe une place particulière parmi les politiques de prévention mais aussi sur le plan de la responsabilité. Les services de psychiatrie sont de plus en plus sollicités pour non seulement prévenir le passage à l'acte mais également pour médicaliser les suites de certains comportements déviants (automutilations, addictions, formes de sexualité hors «normes»). Doit-on (peut-on) les considérer comme l'expression absolue d'une liberté individuelle (celle de mettre fin à ses jours et de disposer de son corps) ou au contraire comme l'acte d'un individu fragile, malade (pour ne pas dire plus totalement responsable de l'ensemble de ses actes) que la puissance publique aurait dû protéger par des moyens adaptés? Pour répondre à cette question, le juriste doit être en mesure de qualifier très précisément les obligations à la charge de chacun car c'est de celles-ci que va découler une éventuelle responsabilité. À l'instar de ce qu'a décrit Émile Durkheim, en 1897, pour le droit, tous les suicides ne sont pas identiques. Sans prétendre dresser ici une typologie exhaustive des situations juridiques possibles, il convient néanmoins de distinguer ce qui peut être considéré comme un autopréjudice (donc excluant toute idée d'indemnisation) et ce qui met en évidence un dysfonctionnement institutionnel. La séparation entre les deux varie principalement en fonction de la prévisibilité du passage à l'acte et de la nature du lien qui unit la victime avec l'institution. La connaissance précise du risque suicidaire modifie en effet les obligations à la charge des «tiers » désignés pour surveiller la personne signalée comme vulnérable. Lorsqu'un individu est considéré comme libre de ses mouvements, la faute d'une tierce personne est particulièrement difficile à démontrer car elle reviendrait à nier l'existence de cette liberté. À l'inverse, si un texte juridique a désigné la personne comme devant bénéficier d'une attention particulière et organise une surveillance de sa liberté d'aller et de venir, la situation juridique n'est plus la même.

9. CAA Lyon, $S^{t e ́}$ AXA France IARD, 12 janvier 2012, n 11 LYoo 331 : «Considérant qu'il résulte de l'instruction, et en particulier du rapport d'expertise déposé le 10 août 2012, qu'après la sortie de M. A du centre hospitalier spécialisé de La Charité sur Loire du 9 janvier 2004, en fugue [le terme est discutable lorsque le patient n'est pas en soins sous contrainte], et donc sans aucune ordonnance de sortie, aucune tentative n'a été entreprise par l'équipe soignante pour tenter de rétablir un lien thérapeutique avec ce patient, souffrant d'une pathologie psychotique lourde et chronique et dont les antécédents montraient de nombreux épisodes délirants aigus avec en association des troubles du comportement à plusieurs reprises, graves, par lesquels il se mettait lui-même en danger et qui pouvaient également représenter une certaine dangerosité pour autrui, et alors qu'il était évident qu'il se trouvait sans traitement; qu'ainsi, en n'engageant aucune tentative de rétablissement d'un lien thérapeutique avec M. A et en ne prévenant pas son entourage, alors même qu'ils connaissaient sa vulnérabilité et sa dangerosité en situation de rupture thérapeutique et médicamenteuse, les services du centre hospitalier spécialisé de La Charité sur Loire dans lequel l'intéressé était hospitalisé en service libre, et alors même qu'une telle hospitalisation ne constitue pas une méthode thérapeutique créant un risque spécial pour les tiers, ont commis une faute de nature à engager la responsabilité de cet établissement ».

10. Art. L. 3212-1 CSP. 
En matière de psychiatrie, la décision d'hospitalisation est incontestablement attentatoire à la liberté d'aller et de venir. Il peut s'agir d'une décision volontaire dans le cadre d'une hospitalisation libre (art. L. 3211-2 CSP) ou d'une contrainte dans le cadre d'une hospitalisation d'office (art. 3213-1 sq. CSP) et d'une hospitalisation à la demande d'un tiers (art. L. 3212-1 sq. CSP). À elle seule, l'hospitalisation ne suffit à pas à présumer la faute du service en cas de suicide d'un patient, même si elle est sous contrainte. Les juges vont certes partir du principe qu'il pèse sur le service une obligation de moyens visant en particulier à diagnostiquer les troubles de l'individu pris en charge et les risques de passage à l'acte. Par contre, la faute ne sera constituée que si le comportement des agents et / ou l'organisation du service étaient manifestement inadaptés à la situation.

Dans une affaire jugée en avril 2013, la cour administrative d'appel de Bordeaux est ainsi venue rappeler que le suicide pouvait être un acte personnel d'un individu y compris lorsqu'il souffrait de troubles mentaux et que toute mort n'ouvrait pas droit à une indemnisation systématique $^{11}$. La solution n'allait pourtant pas de soi, surtout lorsque le patient est vulnérable. Il n'est cependant pas question de faire peser sur l'établissement de santé une présomption de responsabilité pour chaque tentative de suicide. Lorsque la victime ou ses proches souhaitent obtenir réparation des préjudices subis, ils sont tenus de démontrer à la fois la faute de l'établissement et l'existence d'un lien de causalité entre le fait générateur du dommage et les préjudices invoqués. La vulnérabilité du patient crée certes des obligations particulières à l'égard de l'établissement mais ne conduit pas à une présomption de responsabilité.

La cour administrative d'appel a ainsi refusé de reconnaître l'existence d'une faute lors du suicide d'un patient pourtant doublement contraint puisqu'hospitalisé suite à la demande d'un tiers (donc incapable de consentir aux soins) et en plus placé en chambre d'isolement. Ce patient avait été admis en soins psychiatriques suite à une alcoolisation majeure. Ce type de demande de prise en charge vise à faire prendre conscience au patient qu'il souffre d'une pathologie mentale et qu'il a besoin de soins. En l'espèce, celui-ci est resté près d'un mois en hospitalisation complète avant de bénéficier d'une sortie d'essai de dix jours. Deux jours plus tard, le patient se ré-alcoolisait très fortement. Ce comportement a immédiatement entraîné sa réhospitalisation. Le lendemain, ledit patient « fuguait» avant d'être récupéré avec un taux d'alcoolémie de cinq grammes par litre de sang. À son retour dans l'établissement, il était rapidement placé en chambre d'isolement suite à une décision individuelle du psychiatre. Un jour plus tard, le patient s'était donné la mort. Sa fille estimait que l'établissement avait commis une faute dans la surveillance de son père. Cette situation pourrait encore parfaitement se rencontrer avec le nouveau cadre législatif de 2011. Seule la qualification juridique des différentes étapes de sa prise en charge aurait évolué. Dans cette situation, la prise en charge du patient débuterait par une période d'observation de soixante-douze heures visant à déterminer si le patient est bien incapable de consentir aux soins et s'il a besoin d'une prise en charge. Il serait par conséquent examiné deux fois durant la phase préliminaire d'observation (certificat des vingt-quatre heures et des soixante-douze heures), à l'issue de laquelle un psychiatre de l'établissement proposerait au directeur une modalité de prise en charge adaptée au profil et au comportement du patient: hospitalisation complète ou programme de soins. Lorsque l'état du patient le justifie, il est possible de le placer dans une chambre spécifique, adaptée à son état (chambre d'isolement, chambre de soins intensifs...). Ce type de chambre est considéré comme une modalité de soins.

En l'espèce, pour savoir si la responsabilité de l'établissement pouvait être retenue, les juges bordelais ont cherché à établir à la fois la prévisibilité du passage à l'acte et l'existence d'une éventuelle faute dans l'organisation ou le fonctionnement du service. Refusant de présumer la faute de l'établissement, la cour a préféré procéder à une analyse précise de la situation en s'appuyant sur la chronologie des faits et sur l'analyse du fonctionnement du service par une consultation du dossier médical du patient. Cette analyse minutieuse du déroulement du séjour à l'hôpital, et en particulier des heures qui ont précédé la mort du patient, a permis de rappeler que bien que le patient soit privé de liberté de circulation et qu'il soit sous l'emprise de l'administration, cette dernière n'avait qu'une obligation de moyens à son endroit. Il n'a peut-être pas choisi d'être placé en chambre d'isolement mais il pouvait sous certaines conditions être considéré comme le seul responsable d'un acte auto-agressif.

Le juge administratif rappelle à juste titre un principe important, à savoir que tous les patients hospitalisés sous contrainte ne sont pas présumés suicidaires ou dangereux. Chaque situation est unique et suppose une prise en charge individualisée. Il existe certes des protocoles concernant l'usage de la contrainte et le placement à l'isolement mais, à chaque fois, il importe à l'équipe médicale de prendre des mesures de précaution adaptées à l'état du patient. Dans cette affaire rien ne laissait présager un passage à l'acte suicidaire imminent. Au contraire, l'équipe soignante a usé de la contrainte de manière proportionnée. Selon les juges, le patient

[...] s'était montré calme et coopérant tant durant la matinée que l'après-midi, [...] il a pris ses traitements, à 16 heures un Valium 10 milligrammes et à 17 heures un Tercian de 50 milligrammes; [...] il n'avait exprimé ni idées noires, ni idées suicidaires; [...] il a été vu toutes les heures de 15 heures à 18 heures par un infirmier. 
L'expert a d'ailleurs conclu que «cette surveillance et ces soins étaient adaptés à son état; que ni son comportement, ni sa pathologie n'impliquait l'intervention de mesures particulières destinées à prévenir une tentative de suicide». Par conséquent, la Cour a considéré que le suicide n'était pas dû à une faute liée à un acte de prévention, de diagnostic ou de soin et que par conséquent les ayants droit de la victime ne pouvaient prétendre à une indemnisation au titre de la solidarité nationale (art. L. 1142-1 CSP) ${ }^{12}$.

Il est important que le juge puisse se prononcer sur l'existence d'une faute dans l'organisation ou le fonctionnement du service et que la jurisprudence ne bascule pas vers une responsabilité sans faute dans ce type de situation. En conservant une fonction à la faute, on évite le développement de pratiques niant la liberté individuelle ou réduisant exagérément les libertés fondamentales des patients. $\mathrm{Si}$ tous les patients étaient considérés systématiquement comme fragiles, vulnérables et susceptibles de passer à tout moment à l'acte, les établissements accueillant des personnes en soins sous contrainte seraient alors tenus à une forme d'obligation de résultat. Dans cette affaire, la fille du défunt estimait que l'établissement aurait dû condamner la porte de communication entre la chambre et le cabinet de toilette, ce qui aurait peut-être évité le suicide. Lorsque l'on consulte les recommandations de la Haute autorité de santé, il est certes possible pour un établissement de prendre des mesures exceptionnelles de ce type, mais celles-ci doivent être justifiées par un risque imminent. Il n'est pas question de permettre l'organisation d'un système panoptique privant l'ensemble des patients de toute intimité. Le principe de précaution ne doit pas conduire à l'ultra-sécurisation des locaux. De plus en plus, comme c'est le cas pour les mesures de police classiques, les juges insistent sur le caractère strictement nécessaire et proportionné des mesures de contrainte. Prendre en charge, sous la contrainte, des patients souffrant de troubles mentaux est une activité difficile qui suppose une attention constante et une organisation optimale; les juges rappellent fort à propos l'importance de la place de la faute en matière de responsabilité et surtout le fait que l'administration ne peut pas être responsable de tout.
Ce rappel régulier est primordial car, à défaut de pouvoir expliquer précisément les causes exactes d'un passage à l'acte suicidaire, les proches essayent de plus en plus souvent de faire reconnaître la responsabilité de l'administration ${ }^{13}$. Une affaire examinée en 2009 par le Conseil d'État à propos du versement d'une indemnisation de préjudice moral causé par un décès $(5000$ euros versés à la sœur de la victime) a été l'occasion de s'interroger sur le développement de plus en plus fréquent de ce type de recours en recherchant l'existence éventuelle d'une faute de service et celle d'un lien de causalité suffisant entre ladite faute et le décès ${ }^{14}$. La patiente avait été hospitalisée à plusieurs reprises dans un centre hospitalier spécialisé en raison de risques de passage à l'acte suicidaire. Son parcours psychiatrique a débuté en hospitalisation libre, puis, quelques mois plus tard, il a évolué sous le régime de l'hospitalisation sur demande d'un tiers en raison d'un péril imminent. Durant cette hospitalisation sous contrainte, elle avait commis deux tentatives de suicide en deux jours d'intervalle. Malgré ses antécédents, elle est parvenue à sortir du service échappant à la surveillance constante du personnel soignant et a ingéré volontairement un produit détergent caustique, déniché dans un pavillon voisin de l'hôpital. Elle est décédée, cinq mois plus tard, des suites des graves brûlures causées par l'ingestion du produit toxique. Sa famille souhaitait faire reconnaître l'existence d'une faute de l'hôpital. Pour reconnaître l'existence d'une faute, les juges ont tenu compte à la fois des circonstances de l'hospitalisation (demande d'un tiers, incapacité à consentir aux soins), de la personnalité de la patiente, de la prévisibilité d'un nouveau passage à l'acte et de l'organisation interne du service. La conjugaison de toutes ces données permet au juge de considérer qu'une faute de service a été commise. En procédant de la même manière qu'en milieu pénitentiaire ${ }^{15}$, les juges mettent en évidence la défaillance dans la mission confiée à l'institution hospitalière, et estiment qu'un lien de causalité suffisant existe entre la faute et le préjudice subi par la famille. De la même manière, la cour administrative d'appel de Bordeaux vient de juger en $2014^{16}$ que l'établissement de santé ne peut être tenu responsable d'un passage à l'acte qu'il ne pouvait prévoir compte tenu du comportement

12. «Hors le cas où leur responsabilité est encourue en raison d'un défaut d'un produit de santé, les professionnels de santé mentionnés à la quatrième partie du présent code, ainsi que tout établissement, service ou organisme dans lesquels sont réalisés des actes individuels de prévention, de diagnostic ou de soins ne sont responsables des conséquences dommageables d'actes de prévention, de diagnostic ou de soins qu'en cas de faute».

13. État ou établissement public: CE, M. G., 9 mars 2009, $\mathrm{n}^{\circ} 303983$ : «Considérant que, compte tenu des circonstances de son hospitalisation et de la parfaite connaissance qu'avaient les médecins des risques que comportait son état mental, le fait que $\mathrm{M}^{\text {lle }}$ Maggie B ait pu échapper à la vigilance du service où elle était hospitalisée et ait pu mettre fin à ses jours révèle une défaillance dans la surveillance et une faute dans l'organisation du service; que cette faute est directement à l'origine de l'accident qui a entraîné la mort de $\mathrm{M}^{\mathrm{lle}} \mathrm{B}$; qu'elle est de nature à engager la responsabilité de l'établissement hospitalier».

14. Sur la difficile reconnaissance de ce lien, voir CAA Marseille, 12 décembre 2005, nº4Moo471, L'actualité juridique. Droit pénal, 2006, p. 133.

15. CE, Chabba, 23 mai 2003, n 244663: l'administration pénitentiaire est condamnée pour le suicide d'un détenu, le juge estimant qu'il est la conséquence d'« une succession de fautes imputables au service pénitentiaire».

16. CAA Bordeaux, 6 mai 2014, n 12BX02255: "Considérant qu'il résulte de l'instruction que le transfert de Davy Lusignan dans une unité de soins où seraient pratiquées des méthodes plus contraignantes pouvait être envisagé, était même souhaitable et avait été prévu à bref délai; que toutefois, ce transfert, qui aurait été justifié par le traitement de sa maladie, laquelle n'était pas, ainsi qu'il vient d'être dit, caractérisée par des tendances suicidaires, ne présentait pas un caractère d'urgence tel que le fait de ne pas y avoir procédé dans les jours ayant immédiatement suivi l'hospitalisation du patient constituerait un manquement dans la prise en charge médicale de Davy Lusignan ou une faute dans l'organisation ou dans le fonctionnement du centre hospitalier de la Côte Basque; que dès lors et en admettant même qu'un tel transfert aurait diminué d'un quart le risque d'un suicide, $\mathrm{M}^{\mathrm{me}} \mathrm{A}$... n'est pas fondée à soutenir qu'elle devait être indemnisée, ne serait-ce que du fait d'une perte de chance». 
du patient depuis le début de sa prise en charge et que rien n’imposait la modification de sa prise en charge.

\section{Les dommages causés par les patients admis en psychiatrie}

Les dommages causés par les patients souffrant de troubles psychiques sont susceptibles d'engager la responsabilité de l'établissement hospitalier chargé de leur prise en charge. Si le principe reste la responsabilité pour faute, il existe quelques situations dans lesquelles les victimes pourront invoquer le bénéfice de la responsabilité sans faute. Toute la difficulté sera de qualifier au cas par cas la nature des obligations pesant sur l'établissement de santé au moment des faits. Plusieurs situations peuvent ainsi se rencontrer.

\section{A. Les dommages causés par un patient en soins libres}

Le Conseil d'État a par exemple eu l'occasion de se prononcer sur le cas d'un mineur de 17 ans suivi en hôpital de jour par le centre hospitalier de Brive-la-Gaillarde qui avait violemment agressé sa mère lors d'une «crise de démence ${ }^{17}$. Il a logiquement refusé l'application d'un régime de responsabilité sans faute ${ }^{18}$. Le père de l'agresseur et son assurance souhaitaient obtenir une indemnisation des préjudices subis estimant que l'établissement avait failli à son obligation de garde et de surveillance d'un patient doublement signalé puisque mineur et malade mental. L'essentiel du débat portait alors sur le régime de responsabilité à mettre en œuvre. Devait-on comme les juges du fond admettre un recours à la responsabilité sans faute ou au contraire exiger la démonstration de l'existence d'une faute de service et d'un lien de causalité pour accorder le versement de dommages-intérêts. Pour écarter le raisonnement suivi par la cour administrative d'appel, le Conseil d'État a pris soin de qualifier précisément le régime de l'hospitalisation en hôpital de jour et les circonstances de l'agression. Pour admettre la possibilité d'invoquer un régime de responsabilité sans faute, il convenait de démontrer l'existence de la mise en œuvre d'une méthode dangereuse. Pouvait-on considérer que ce type d'hospitalisation entraînait la reconnaissance d'une mission de garde à la charge du service ${ }^{19}$ ?

Reprenant les critères dégagés en $2010^{20}$, le Conseil précise que le mode de prise en charge «n'a pas eu pour effet de transférer à cet établissement la responsabilité d'organiser, diriger et contrôler la vie de ce mineur qui se trouvait, à la sortie de l'hôpital de jour, sous la garde légale de son père». Il n'y a pas eu création d'une obligation de garde du patient mineur qui reste durant toute la période de soin, en particulier lorsqu'il se trouve en dehors de l'établissement, sous la responsabilité des personnes exerçant l'autorité parentale. Cela ne signifie pas qu'il soit impossible pour les victimes de poursuivre les services de santé, mais cela devra alors se faire sur le terrain de la faute. Sur cette question, le Conseil d'État recherche si les soignants ont prescrit des traitements correspondant à la pathologie diagnostiquée, et si ces derniers étaient conformes aux données de la science. Pour qu'une faute puisse être retenue contre le service, il fallait aussi déterminer si la prévisibilité de son comportement agressif était objectivement quantifiable. Les soins proposés au mineur correspondaient, pour le juge, à son état psychologique et au respect de ses droits fondamentaux. Le traitement en hospitalisation de jour, avec retour quotidien dans la famille, avait été institué en août 2000 après une période d'essai concluante. De plus, cela faisait quatre mois qu'il se déroulait sans incident et que l'état du patient était incontestablement en voie d'amélioration puisqu'aucun geste d'agressivité n'avait été signalé au service par les proches du patient. La responsabilité pour faute reste donc le principe et la théorie du risque l'exception. Ce choix du juge administratif est lourd de conséquence pour les proches des patients mineurs (et sous certaines conditions majeures) qui se retrouvent associés à la réussite de la prise en charge du fait d'une forme d'obligation de vigilance, voire de surveillance et d'alerte.

La position retenue dans cet arrêt du 17 février 2012 est importante car elle permet d'opérer une distinction entre les divers modes de prise en charge des patients. Les obligations pesant sur les services de santé sont déterminées en fonction du statut du patient (hospitalisation libre et hospitalisation sous contrainte), de la pathologie

17. CE, Société MAAF assurances, 17 février 2012, nº 334766

18. CE, sect., Hôpital psychiatrique départemental de Rennes c. Dame Clotault, 30 juin 1978, Recueil Lebon, p. 289; Dalloz, 1979, IR 112, obs. F. Moderne; L'actualité juridique. Droit administratif, $\mathrm{n}^{\circ} 11,1978$, p. 590 et p. 556, chron. O. Dutheillet de Lamothe et Y. Robineau; Revue de droit sanitaire et social, 1979, p. 379, note F. Moderne; CE, C ${ }^{\text {ie }}$ d'assurances l'Europe et Trochon, ${ }^{\text {er }}$ février 1980, mentionné aux tables du Recueil Lebon, p. 889; L'actualité juridique. Droit administratif, no 6, 1980, p. 376; Revue de droit sanitaire et social, 1980, p. 495, obs. J.-M. de Forges; CE, Bourgeois, 26 octobre 1984, Revue du droit public, 1985, p. 1375, obs. Y. Gaudemet.

19. CE, GIE Axa Courtage, 11 février 2005, n² 252169, Recueil Lebon, p. 45; L'actualité juridique. Droit administratif, n 12,2005 , p. 663, chron. C. Landais, F. Lenica; Dalloz, 2005, p. 1762, note F. Lemaire; L'actualité juridique. Droit pénal, 2005, p. 198, obs. C. S. Enderlin; Revue française de droit administratif, $\mathrm{n}^{\circ}$ 3, 2005, p. 595-601, concl. C. Devys et p. 602-607, note P. Bon; Revue de droit sanitaire et social, 2005, p. 466, note D. Cristol; Revue trimestrielle de droit civil, 2005, p. 585, obs. J. Hauser; La semaine juridique, éd. G, 2005, II, 10070, concl. C. Devys, note M.-C. Rouault; La semaine juridique, édition administrations et collectivités territoriales, $\mathrm{n}^{\circ} 11,14$ mars 2005 , chron. $\mathrm{n}^{\circ} 1132$, note J. Moreau; Les petites affiches, $\mathrm{n}^{\circ} 108,2005$, p. 8, note E. Matutano; Responsabilité civile et assurances, $\mathrm{n}^{\circ} 6,2005$, comm. n 192 , note C. Guettier; La semaine juridique, éd. G, 2005, I, 149, nº 4, obs. G. Viney; Droit de la famille, 2005, comm. n 173, note X. Dupré de Boulois; CE, Ministre de la justice, 17 décembre 2008 , nº 301705; CE, Département des Côtes d'Armor, 26 mai 2008, nº 290495.

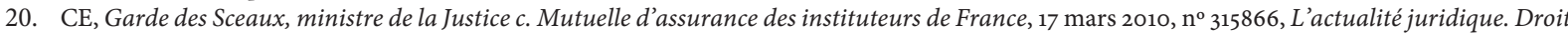
administratif, $\mathrm{n}^{\circ}$ 11, 2010, p. 580 et $\mathrm{n}^{\circ}$ 21, 2010, p. 1209, concl. C. Roger-Lacan; Revue de droit sanitaire et social, 2010, p. 568, obs. D. Cristol. 
mais aussi des lieux et des circonstances du dommage. À l'instar de la méthode retenue pour les autres services publics, le juge administratif se doit d'appréhender chaque situation selon des critères prévisibles. C’est ainsi que:

[...] l'hôpital de jour, qui est un mode de prise en charge hospitalier destiné à assurer des soins polyvalents mis en œuvre par une équipe pluridisciplinaire en un lieu ouvert à la journée selon une périodicité déterminée pour chaque patient, ne constitue pas une méthode thérapeutique créant un risque spécial pour les tiers susceptible d'engager sans faute la responsabilité de l'administration.

Dans l'intérêt du patient et pour le bon fonctionnement du service, la notion de garde et de surveillance n'a pas vocation à s'étendre démesurément. C'est donc à dessein que le Conseil d'État a réduit le champ de la responsabilité sans faute.

L'instauration par la loi du 5 juillet 2011 du programme de soins comme alternative à l'hospitalisation complète en matière de soins sous contrainte ne manquera pas d'entraîner des recours en responsabilité du fait des dommages causés par les patients "contraints». Le Conseil constitutionnel venant de déclarer le dispositif conforme à la Constitution ${ }^{21}$, certains patients seront désormais placés sans leur consentement en hôpital de jour. Cette prise en charge ne créera pas d'obligation de résultat à la charge du service mais une simple obligation de moyen. Les hôpitaux ne devraient donc pas avoir à supporter seul le poids d'une socialisation du risque. Le Conseil d'État refuse en effet d'organiser une présomption de dangerosité pesant sur le malade mental et surtout de qualifier l'hospitalisation de jour de méthode dangereuse ${ }^{22}$. Cette décision protège indirectement les droits du patient en évitant que les services renoncent, par précaution, à certaines modalités de soins. La généralisation d'une responsabilité sans faute fondée sur une conception extensive de la garde aurait en effet conduit les services à se surprotéger pour éviter le risque contentieux. Peut-on pour autant considérer que l'hospitalisation en hôpital de jour est assimilable à une hospitalisation libre ${ }^{23}$ ? Cela n'est pas certain, il faudra encore distinguer entre les activités au sein de l'établissement (où l'usage de la contrainte peut modifier l'étendue des obligations et le champ de la faute) et les faits et gestes du patient à l'extérieur.

\section{B. Les dommages causés par un (ex-)patient en soins sous contrainte}

Cette seconde hypothèse peut se rencontrer dans plusieurs situations. La plus courante est celle où le patient se trouve en dehors de l'établissement sans surveillance constante d'un personnel suite à une décision administrative (programme de soins ou sortie de courte durée de moins de quarante-huit heures). La deuxième situation est celle où le patient est en fugue et la troisième se rencontre lorsqu'il commet un dommage à un tiers (visiteurs ou autres patients à l'intérieur de l'établissement).

En ce qui concerne la fugue d'un patient ${ }^{24}$, le juge administratif adopte une position désormais classique. Il commence par décomposer avec précision chaque étape ayant conduit au dommage afin de pouvoir imputer à chaque acteur concerné par la prise en charge psychiatrique sa juste part de responsabilité. L'objectif principal est de déterminer si le patient est en «fugue» ou en rupture de programme de soins. Les conséquences de cette qualification sont importantes car le régime de responsabilité qui en découle ne sera pas le même et l'imputabilité de la responsabilité pourra également varier. L'analyse peut s'avérer délicate dans les affaires d'agression faisant suite à une «sortie» donnant lieu à une agression plusieurs jours après que le patient ait échappé à la surveillance du service. Cette situation peut parfois s'avérer complexe. Ce fut le cas dans une affaire largement médiatisée où le patient, en fugue depuis dix-huit jours, a tué à coups de hache le compagnon octogénaire de sa grand-mère. La famille de la victime souhaitait obtenir une indemnisation des préjudices subis et tentait de faire reconnaître la responsabilité conjointe de l'hôpital et de l'État. La principale difficulté de l'affaire était de déterminer si l'autorité de police à l'origine de la mesure de soins et prévenue de la fugue pouvait être

21. CC, déc. 2012-235 QPC du 20 avril 2012: «11. Considérant, en premier lieu, que, lorsqu'une personne faisant l'objet de soins psychiatriques sans son consentement n'est pas prise en charge sous la forme d'une hospitalisation complète, un "programme de soins" est établi par un psychiatre de l'établissement; que l'avis du patient est recueilli préalablement à la définition et avant toute modification de ce programme, à l'occasion d'un entretien au cours duquel il reçoit l'information prévue à l'article L. 3211-3 et est avisé des dispositions de l'article L. 3211-11; que le second alinéa de l'article L. 3211-11 du code de la santé publique dispose que, lorsque le psychiatre constate que la prise en charge sous la forme ambulatoire ne permet plus, notamment du fait du comportement de la personne, de dispenser les soins nécessaires à son état, il "transmet immédiatement au directeur de l'établissement d'accueil un certificat médical circonstancié proposant une hospitalisation complète"; que le dernier alinéa de l'article L. 3212-4 et le paragraphe III de l'article L. 32133 fixent les modalités selon lesquelles une prise en charge au titre du $2^{\circ}$ de l'article L. $3211-2-1$ peut être modifiée à cette fin;/12. Considérant qu'il résulte de ces dispositions qu'en permettant que des personnes qui ne sont pas prises en charge en "hospitalisation complète" soient soumises à une obligation de soins psychiatriques pouvant comporter, le cas échéant, des séjours en établissement, les dispositions de l'article L. 3211-2-1 n'autorisent pas l'exécution d'une telle obligation sous la contrainte; que ces personnes ne sauraient se voir administrer des soins de manière coercitive ni être conduites ou maintenues de force pour accomplir les séjours en établissement prévus par le programme de soins; qu'aucune mesure de contrainte à l'égard d'une personne prise en charge dans les conditions prévues par le $2^{\circ}$ de l'article L. 3211-2-1 ne peut être mise en cuvre sans que la prise en charge ait été préalablement transformée en hospitalisation complète; que, dans ces conditions, le grief tiré de la violation de la liberté individuelle manque en fait» (nous soulignons).

22. CE, Thouzellier, 3 février 1956, Recueil Lebon, p. 49; Dalloz, 1956, p. 597, note J.-M. Auby.

23. Responsabilité pour faute: CE, Centre psychothérapeutique de la Nièvre, 30 juin 1978, nº 98940, 04985, Recueil Lebon, p. 287; Dalloz, 1979, IR 113, obs. F. Moderne.

24. CAA Marseille, $\mathbf{1}^{\text {er }}$ octobre 2009, $\mathrm{n}^{\circ}$ o7MAo3488. 
considérée comme responsable des dommages causés ou si la responsabilité pouvait être imputée à l'hôpital qui n'avait pas empêché qu'un tel patient soit dehors.

Le 19 juin 2007, le tribunal administratif de Marseille a déclaré l'État (le préfet, autorité de police) seul responsable des dommages causés par le patient. La cour administrative d'appel prend une décision radicalement différente mais guère plus convaincante. Selon elle, il convient d'analyser précisément, en fonction de la législation en vigueur à l'époque, la situation et le déroulement des faits et de rechercher l'existence d'un lien de causalité unique entre le fait dommageable et les préjudices. Cette méthode permet d'écarter toute responsabilité de l'État, y compris pour faute, dans l'organisation des forces de l'ordre chargées de retrouver le patient en fugue, dont la dangerosité était pourtant connue: l'auteur du dommage avait été arrêté par la police pour une série d'actes de violence et une tentative d'assassinat. Reconnu pénalement irresponsable (art. L. 122-1 du Code pénal), il avait été hospitalisé d'office ${ }^{25}$. Durant son hospitalisation, dans un souci thérapeutique, le préfet lui a accordé sept sorties d'essai à temps complet et à durée déterminée ${ }^{26}$. Cette décision d'assouplir la mesure de police repose toujours sur une proposition écrite et motivée d'un psychiatre de l'établissement (engagement possible de la responsabilité de l'État, seule autorité décisionnaire). C'est justement durant une de ces périodes de sortie que le patient a quitté précipitamment le service sans que le personnel présent ne parvienne à le retenir ${ }^{27}$. Selon la cour administrative, les antécédents du patient auraient dû entraîner une surveillance renforcée de la part de l'établissement. Cette carence démontre un «fonctionnement défectueux du service de nature à engager la responsabilité de l'hôpital». Sept jours plus tard, le patient toujours en fugue se rend chez sa victime et a avec elle une altercation qui nécessite l'intervention de la police. Le patient n'est pas appréhendé (faute de la police?) et revient sur les lieux onze jours après pour assassiner la victime: acte pour lequel il sera de nouveau déclaré pénalement irresponsable.

L'enchevêtrement des faits rendait l'affaire confuse et a amené les juges du fond à deux interprétations différentes. Le tribunal administratif estimait que le régime de la responsabilité sans faute organisé pour les sorties thérapeutiques pouvait s'appliquer et peser sur l'État qui par le biais de son représentant avait assoupli les conditions d'hospitalisation. La cour administrative d'appel, quant à elle, préfère retenir le régime de responsabilité pour faute de l'établissement de santé à qui le préfet avait confié la prise en charge en considérant que l'on se trouvait en situation de «fugue» et que par conséquent le préfet ne pouvait être tenu pour responsable de l'agression. Il arrive parfois qu'en privilégiant une autre approche du lien de causalité le juge retienne la responsabilité des forces de l'ordre qui ne sont pas parvenues à retrouver le fugueur (ou l'évadé) et à empêcher la survenance des préjudices ${ }^{28}$. Tout dépend de savoir qui a commis le dommage: l'usager de la mesure de police, le patient soigné et pris en charge par l'institution hospitalière ou le fugueur échappant à ses poursuivants.

Si la même affaire se renouvelait aujourd'hui, c'est-àdire en application du régime issu de la réforme de 2011, la détermination du régime de responsabilité ne serait pas beaucoup plus simple. Dans la mesure où le patient fait l'objet d'une prise en charge à la suite d'une demande d'un tiers $^{29}$ et s'il cause un dommage en dehors de l'établissement, trois situations sont alors possibles. La première résulte de l'application de l'article L. 3211-11-1 du Code de la santé publique ${ }^{30}$. Dans ce cas le patient est considéré

25. Ce type de décision de police administrative est susceptible d'engager la responsabilité de l'État. Selon le Tribunal des conflits, le juge judiciaire est compétent pour statuer sur la réparation du préjudice subi du fait d'un arrêté de placement d'office: TC, Préfet de la région Île-de-France, 17 février 1997, Recueil Lebon, p. 525. Voir également, CE, 5 juin 1996, n 155138 : «lorsqu'il admet ou maintient dans son établissement un malade dont l'autorité compétente a ordonné le placement d'office ou le maintien en placement d'office, le directeur d'un hôpital psychiatrique se borne à exécuter cet ordre et ne prend pas lui-même une nouvelle décision susceptible de faire l'objet d'un recours pour excès de pouvoir ".

26. Avec la loi de 2011, le patient aurait été placé en programme de soins par le préfet sur proposition d'un psychiatre de l'établissement.

27. Possibilité de rechercher la faute de l'établissement au moment de la fugue: responsabilité pour faute, y compris pour un patient en hospitalisation libre, voir CAA Nancy, CH Brienne-le-Château, 12 novembre 2009, nº $08 \mathrm{NC} 00590$ : «Considérant, d'une part, que $\mathrm{M}^{\mathrm{lle}}$ Sabiha B, âgée de trentedeux ans, qui avait été admise au centre hospitalier de Brienne-le-Château en service libre le 30 décembre 2003, est sortie de l'établissement le 29 juillet 2004 vers 10h30; qu'à cette date, il n'est pas contesté qu'elle ne présentait pas de tendances suicidaires, ni même de syndrome dépressif; que même si elle avait montré une agressivité envers les autres patients les 17 et 27 juillet précédents, agressivité dont elle était coutumière, elle n'a pas manifesté, dans les heures qui ont précédé sa fugue, un comportement particulier nécessitant un renforcement de la surveillance alors exercée selon les seules exigences du placement libre; que, quand bien même elle avait précédemment fugué les 2 février, 9 avril et 30 juin 2004 , la circonstance qu'elle ne faisait pas l'objet d'une surveillance constante le 29 juillet 2004, qu'il n'y avait pas lieu de mettre en œuvre, ne constitue pas, dans les circonstances de l'espèce, une faute dans l'organisation et le fonctionnement du service public hospitalier; / Considérant, d'autre part, que l'absence de $\mathrm{M}^{\mathrm{Ile}} \mathrm{B}$ a été découverte dès 11h3o lors de la distribution des médicaments aux patients; que des recherches, certes infructueuses, furent immédiatement engagées au sein de l'établissement et dans les villages situés à proximité; qu'il résulte de l'instruction que dès que la présence de $\mathrm{M}^{\mathrm{Hl}} \mathrm{B}$ au port de Dienville fut effectivement signalée après 13h30, deux agents de l'hôpital se sont rendus promptement sur place pour poursuivre les recherches; qu'ainsi, ni le délai mis pour découvrir la disparition de la victime, ni le temps consacré aux démarches entreprises pour la retrouver ne peuvent davantage être regardés comme constitutifs d'une faute de nature à engager la responsabilité du service hospitalier ».

28. CE, Ramade, 10 mai 1985, n 48517, Recueil Lebon, p. 147 ; L'actualité juridique. Droit administratif, 1985, p. 568, note L. R.; Droit administratif, $\mathrm{n}^{\circ} 10,1985$, comm. no 327 .

29. Art. L. 3212-1 sq. CSP.

30. Art. L. 3211-11-1 CSP modifié par la loi n²013-869 du 27 septembre 2013: «Afin de favoriser leur guérison, leur réadaptation ou leur réinsertion sociale ou si des démarches extérieures sont nécessaires, les personnes faisant l'objet de soins psychiatriques en application des chapitres II et III du présent titre ou de l'article 706-135 du code de procédure pénale sous la forme d’une hospitalisation complète peuvent bénéficier d'autorisations de sortie de courte durée : $1^{\circ}$ Sous la forme de sorties accompagnées n'excédant pas douze heures. Les personnes malades sont accompagnées par un ou plusieurs membres du personnel de l'établissement d'accueil, par un membre de leur famille ou par la personne de confiance qu'elles ont désignée en application de l'article L. 1111- 6 du présent code, pendant toute la durée de la sortie;/ $2^{\circ}$ Sous la forme de sorties non accompagnées 
comme continuant à être hospitalisé en hospitalisation complète, y compris lorsqu'il est accompagné pendant moins de douze heures par un proche (dont le statut est alors analogue à celui de collaborateur du service) ou qu'il bénéficie d'une sortie non accompagnée de moins de quarante-huit heures ${ }^{31}$. La victime devrait alors démontrer l'existence d'une faute de service si elle souhaite engager la responsabilité de l'établissement.

La deuxième situation est sans doute la plus inédite car elle se rencontrera lorsque le patient en programme de soins est à l'origine d'un dommage causé à un tiers. Dans ce cas de figure, il est fort probable que le juge retienne un régime de responsabilité sans faute fondé sur la notion de «risque» ou d'aléa psychiatrique ${ }^{32}$. La troisième situation pourrait se rencontrer lorsque le patient est en fugue et qu'il a donc échappé à la surveillance du service. Le régime de la responsabilité pour faute a alors toute sa place, sachant qu'il est loisible au juge d'inverser la charge de la preuve en demandant à l'établissement de démontrer qu'il n'a commis aucune faute ayant permis ou facilité la fugue du patient. La victime se contenterait alors de démontrer la réalité des préjudices subis et l'existence d'un lien de causalité.

$\mathrm{Si}$, par contre, la prise en charge psychiatrique résulte d'une mesure de police administrative spéciale ${ }^{33}$, l'engagement de la responsabilité est un peu plus complexe car deux institutions sont concernées par la mesure. D'un côté l'autorité de police et de l'autre l'établissement de santé. Lorsque le patient «fugue», il est fort probable que les juges continuent à appliquer la jurisprudence issue de la loi de 1990. Par contre, pour les programmes de soins et les sorties accompagnées, la situation sera plus délicate car la décision administrative est prise par le préfet mais toujours sur la base d'une proposition faite par un psychiatre de l'établissement d'accueil. Par exemple pour une sortie d'un patient en hospitalisation complète, le préfet va tacitement consentir à la sortie. Son silence vaut en effet acceptation de la sortie et donc du «risque». Il n'est donc pas vraiment surprenant que face à ce type de demande, les services de la préfecture ou de l'ARS demandent au psychiatre d'être particulièrement précis dans l'avis qu'il rend préalablement à toute sortie ${ }^{34}$. En effet, le nouveau dispositif instauré par le législateur rend beaucoup plus difficile la possibilité pour un préfet de s'opposer à une sortie de courte durée dès l'instant où un psychiatre de l'établissement estime qu'elle est possible et qu'il est prêt à l'écrire. Enfin, lorsque le patient est en programme de soins arrêté par le préfet et qu'il commet un dommage à l'extérieur, il est fort probable que la victime cherche à démontrer que le contenu dudit programme était manifestement inadapté au patient. Dans ce cas, c'est la responsabilité de l'établissement qui sera selon toute vraisemblance recherchée.

\section{L'atteinte aux droits fondamentaux de la personne accueillie dans un service de psychiatrie}

Le recours en responsabilité est souvent un moyen pour la victime de faire reconnaître l'existence d'un droit subjectif. La recherche d'une indemnisation permet certes de réparer le préjudice subi mais également de faire constater que l'institution a dysfonctionné. Ce n'est plus alors uniquement le patient qui saisit le juge mais l'usager du service, voire la personne humaine. À titre d'illustration, il est possible d'imaginer les suites en plein contentieux de l'affaire de l'interdiction de la sexualité à l'hôpital de Cadillac $^{35}$. En effet, une personne hospitalisée dans un service de psychiatrie peut comme n'importe quel usager contester la légalité des décisions individuelles prises à son encontre mais également celle des actes réglementaires organisant le service. S'il parvient à démontrer qu'une décision illégale lui cause un préjudice, il est en droit d'en demander l'indemnisation, sachant que toute illégalité est constitutive d'une faute.

\section{A. Repenser les règles de fonctionnement des services de psychiatrie afin de respecter les personnes prises en charge}

Les conditions d'hospitalisation dans les établissements de santé mentale ne sont pas toujours très respectueuses des libertés fondamentales des patients. La protection

d'une durée maximale de quarante-huit heures. / L'autorisation de sortie de courte durée est accordée par le directeur de l'établissement d'accueil, après avis favorable d'un psychiatre de cet établissement ».

31. Dans ce cas, il n'est pas possible d'appliquer la jurisprudence relative à la responsabilité sans faute qui a été étendue aux dommages causés aux tiers par des malades faisant l'objet d'un placement familial surveillé: CE, M ${ }^{\text {me }}$ Piollet et M. Anson, 13 mai 1987, Recueil Lebon, p. 172; Dalloz, 1987, IR 158; L'actualité juridique. Droit administratif, no 7-8, 1987, p. 489 et p. 454, obs. M. Azibert et M. Boisdeffre.

32. CE, sect., Département de la Moselle, 13 juillet 1967, Recueil Lebon, p. 341; Dalloz, 1967, p. 675, note F. Moderne; Gazette du palais, 1968, 1, p. 223; Revue du droit public, 1968, p. 391, note M. Waline; L'actualité juridique. Droit administratif, 1968, p. 419, note J. Moreau; Revue de droit sanitaire et social, 1968, p. 108, obs. J. Imbert; TA Pau, 18 mars 1964, Sempé, Dalloz, 1965, p. 312, note F. Moderne. Voir P. Bon, «La responsabilité des services publics utilisant des méthodes libérales », Revue française de droit administratif, 1984, p. 141.

33. Art. L. 3213-1 sq. CSP.

34. Art. L. 3211-11-1 CSP: «Dans le cas où la mesure de soins psychiatriques a été prise en application du chapitre III du présent titre, le directeur de l'établissement d'accueil transmet au représentant de l'État dans le département les éléments d'information relatifs à la demande d'autorisation, comportant notamment l'avis favorable du psychiatre mentionné au quatrième alinéa du présent article, au plus tard quarante-huit heures avant la date prévue pour la sortie. Sauf opposition écrite du représentant de l'État dans le département, notifiée au plus tard douze heures avant la date prévue, la sortie peut avoir lieu. Le représentant de l'État ne peut imposer aucune mesure complémentaire».

35. CAA Bordeaux, M. Claude X c. CHS de Cadillac, 6 novembre 2012, n 11BXo179o, Dalloz, 2013, p. 312, obs. F. Vialla; L'actualité juridique. Droit administratif, n², 2013, p. 115, concl. D. Katz; F. Vialla, «Privé de vie privée: “vol retour” au-dessus d'un nid de coucou? ", Revue droit et santé, $\mathrm{n}^{\circ} 52,2013$, p. 141-151. 
de la vie privée à l'hôpital est un sujet complexe en psychiatrie car on a longtemps occulté ce point en considérant que le traitement de la pathologie justifiait une surveillance panoptique. Il est indispensable pour une institution accueillant, parfois pour de longues durées, des personnes vulnérables d'adopter des règles équilibrées permettant de protéger les droits de chacun (patients, proches, soignants). Après avoir longtemps ignoré ce type de recours, le juge administratif commence à examiner la manière dont les établissements s'organisent au quotidien. Une affaire a récemment fait l'objet de l'attention des médias car elle abordait le sujet tabou de la sexualité des malades mentaux. Usager contraint du CHS de Cadillac, un patient a demandé au directeur d'abroger une partie du règlement intérieur s'appliquant à l'unité chargée de le prendre en charge. Cet acte réglementaire prévoyait que, dans les chambres, "les relations de nature sexuelle ne sont pas autorisées ». Conscient que cette disposition portait atteinte aux libertés individuelles, le texte avait pris soin de la justifier en précisant que «cette interdiction s'impose dans la mesure où les patients d'un établissement psychiatrique sont vulnérables et nécessitent d'être protégés ». Formulée de la sorte, une telle interdiction peut-elle figurer dans un règlement intérieur et, lorsqu'elle est appliquée, est-elle susceptible d'engager la responsabilité de l'établissement? Une telle interrogation pourrait paraître incongrue. Pourtant, lorsque l'on connaît la jurisprudence d'autres services publics accueillant des usagers contraints, comme cela peut être le cas du service public pénitentiaire, il est assez facile de faire un parallèle avec le contentieux de la prise en charge des patients en psychiatrie: droit à la pratique religieuse, droit à des visites familiales ${ }^{36}$. Un usager peu en effet demander à être indemnisé s'il estime que l'application d'un règlement illégal lui a causé un préjudice.

Progressivement, les différentes juridictions commencent à faire émerger un socle commun de libertés fondamentales qu'il convient de préserver. C'est ainsi qu'à la différence d'un usager en hospitalisation libre, les personnes placées en observation durant soixantedouze heures suite à une demande du représentant de l'État (SDRE) ou d'un tiers (SDT), mais surtout celles maintenues en hospitalisation complète à l'issue de cette période initiale ne sont pas assimilables à des «patients classiques». En effet, il leur est difficilement possible de quitter sans surveillance l'établissement, y compris afin de pouvoir exercer leurs droits fondamentaux. Se pose alors inévitablement la question de l'indemnisation éventuelle d'une atteinte disproportionnée à l'un de ces droits. L'hospitalisation complète étant une mesure privative de la liberté d'aller et de venir, l'établissement désigné pour exécuter la mission de service public de soins sous contrainte est tenu d'organiser ses services afin de garantir au mieux le respect des droits de la personne prise en charge. Des règles devront par conséquent être mises en œuvre afin de garantir que quotidiennement ses droits de citoyen, de justiciable, de patient et de personne humaine seront pris en compte. Le législateur le rappelle d'ailleurs expressément en indiquant que:

[...] les restrictions à l'exercice de ses libertés individuelles doivent être adaptées, nécessaires et proportionnées à son état mental et à la mise en œuvre du traitement requis. En toutes circonstances, la dignité de la personne doit être respectée et sa réinsertion recherchée ${ }^{37}$.

En 2011, le Parlement a bien pris soin de rappeler que l'établissement d'accueil doit tout mettre en place pour respecter les droits fondamentaux des personnes prises en charge. Le non-respect d'une telle obligation est par conséquent susceptible d'engager sa responsabilité.

Dans l'affaire de Cadillac, le juge administratif coupe court à tout risque de dérive contentieuse et précise fort justement qu'il n'existe pas un droit à la sexualité et que l'administration ne pourrait être tenue pour responsable de la privation de sexualité d'une personne hospitalisée y compris pour une longue durée ${ }^{38}$. Par contre, lorsqu'un patient souhaite avoir des relations sexuelles avec un partenaire consentant, il n'est a priori pas possible de lui refuser ce droit dès lors que l'acte ne trouble pas l'ordre public ou le bon fonctionnement de l'établissement. Cela signifie par conséquent qu'il n'est pas question d'autoriser à tout moment et n'importe où tout type de comportement individuel, mais il n'est pas non plus possible de priver par principe un patient de sexualité. Le juge administratif résume la situation de la manière suivante:

[...] l'ingérence dans l'exercice du droit d'une personne atteinte de troubles mentaux, hospitalisée sans son consentement, au respect de sa vie privée qui constitue une liberté individuelle et dont le respect de la vie sexuelle est une composante, par une autorité publique, ne peut être légale que si elle répond à des finalités légitimes et qu'elle est adéquate et proportionnée au regard de ses finalités ${ }^{39}$.

Concrètement, pour éviter tout risque contentieux et respecter les droits fondamentaux de la personne en hospitalisation complète, l'établissement de santé va devoir faire face à deux types de situations que l'on rencontre dans tous les lieux privatifs de liberté. La première consiste à devoir gérer les demandes "programmées» de relations intimes formulées par une personne hospitalisée qui souhaite s'isoler pour un moment. Pour répondre à ces

36. TA Grenoble, M. K., 17 octobre 2013, n 1302502, É. Péchillon, «Télévision et repas confessionnel: le principe d’égalité devant le service public en question", L'actualité juridique. Droit pénal, 2014, p. 95; TC, M. C., 14 octobre 2013, n C3918, É. Péchillon, "Travail en prison: une mission de service public visant à la réinsertion du détenu? ", L'actualité juridique. Droit pénal, 2014, p. 47; CE, Garde des Sceaux, ministre de la Justice c. $M$. N et autres, 16 octobre 2013, $\mathrm{n}^{\circ}$ 351115, 351116, 351152, 351153, 351220, 354484, 354485, 354507, 354508, É. Péchillon, «Le culte en détention: une obligation de moyens à la charge de l'administration pénitentiaire », L'actualité juridique. Droit pénal, 2013, p. 685.

37. Art. L. 3211-3 CSP

38. Voir F. Vialla, «Vie affective et sexuelle des personnes handicapées: assistance sexuelle», Dalloz, 2013, p. 714

39. CAA Bordeaux, M. Claude X c. CHS de Cadillac, cons. 6. 
requêtes parfaitement légitimes, il va alors falloir prévoir la création de structures d'accueil spécialisées, proches des unités de vie familiale existant en prison. Ces structures sont notamment indispensables pour les personnes hospitalisées pour une longue durée. Ces demandes supposent que le patient, non seulement fournisse les coordonnées de la personne qui va partager son intimité, mais aussi que les soignants s'interrogent sur l'opportunité de refuser cette intimité. La prise en considération de ce type de demande conduit à un renversement total de la logique institutionnelle traditionnelle qui consistait à ne concevoir l'organisation que sous la forme d'interdits en partant du principe que le patient devait renoncer le temps de son hospitalisation à une large part de sa vie privée.

La seconde situation, plus complexe, suppose de réfléchir à la possibilité d'accorder au patient une part d'intimité et de secret au sein même du service. Être malade psychiatrique suppose-t-il de devoir déclarer non seulement ses pratiques sexuelles ou religieuses mais aussi leur fréquence? C'est alors le statut de la chambre du patient et des relations intimes entre usagers qui sont en cause. Pour protéger les personnes hospitalisées en particulier les plus vulnérables, il importe que l'équipe soignante soit en mesure de connaître l'essentiel de «l'activité» et des faits et gestes des patients au sein de l'établissement, mais pas au prix d'une négation de l'intimité. Une atteinte disproportionnée aux droits fondamentaux de la personne prise en charge est donc susceptible d'engager la responsabilité de l'établissement. Cette affaire de Cadillac est importante car elle a obligé le juge administratif à se prononcer sur la manière dont doivent être rédigés les règlements intérieurs des établissements qui serviront de base à une multitude de décisions individuelles prises durant le séjour qui la plupart du temps ne sont pas en lien direct avec la pathologie.

Un règlement intérieur est un acte réglementaire indispensable à toute institution puisqu'il permet à la fois de garantir la sécurité juridique et d'adapter les normes générales aux circonstances locales particulières. Il serait pourtant question, comme cela est malheureusement encore trop souvent le cas, d'autoriser le chef de service à rédiger une norme locale uniquement en fonction de l'organisation interne des bâtiments ou du nombre de personnels. Ce n'est pas à l'usager, surtout s'il est contraint, de se plier aux contraintes matérielles, mais à l'inverse au chef d'établissement d'adapter sans cesse les règles locales aux normes supérieures (Convention européenne des droits de l'homme, bloc de constitutionnalité) et aux besoins des usagers. Le législateur rappelle ainsi qu'il est hors de question que le règlement intérieur réduise certains droits fondamentaux des patients ${ }^{4}$. Seul le législateur dispose d'un tel pouvoir à condition d'ailleurs de respecter les engagements internationaux pris par la France. Trop souvent le règlement intérieur est rédigé à partir d'un usager idéal-typique conduisant à nier les différences entre individus.

En l'espèce, ce qui était reproché au CHS de Cadillac c'est le fait d'avoir utilisé la technique de l'interdiction générale d'un comportement pour l'ensemble des patients alors que seuls certains d'entre eux auraient mérité, à certaines périodes, de se voir interdire des « relations de nature sexuelle». Outre le fait que le comportement visé par le règlement est ambigu (où commence la sexualité?) et que l'on imagine mal quelles peuvent être les sanctions administratives et disciplinaires prononcées à l'encontre d'une personne se livrant à ces relations, ce type d'interdiction sert surtout à priver le patient de toute intimité et à justifier une forme de panoptique psychiatrique. Autant il est possible d'envisager individuellement et temporairement une surveillance particulière pour certaines pathologies, autant interdire systématiquement un acte autorisé à l'extérieur est difficile à justifier. Si l'acte sexuel s'apparente à un viol ou une agression, le droit pénal permet de sanctionner le coupable et le droit civil d'imposer le versement d'un dédommagement du préjudice. Interdire la sexualité ne l'a jamais empêché et ne permet pas de garantir l'absence de viol et d'agression sexuelle sur des patients vulnérables. Une telle interdiction ne suffit pas non plus à dégager la responsabilité de l'établissement. Aussi les règlements intérieurs doivent-ils aborder de front l'ensemble des demandes formulées par les patients et leurs proches afin de proposer une prise en charge respectueuse des dispositions de la Convention européenne des droits de l'homme (article 8: Droit au respect de la vie privée et familiale) et de favoriser le développement de la réflexion éthique.

C'est sur la base du règlement intérieur éventuellement spécialisé par unités de soins et en conformité avec les normes supérieures que diverses décisions individuelles concernant un patient seront légalement prises. À ce titre, il est parfaitement possible d'interdire, pour raison médicale, à une personne hospitalisée de rencontrer d'autres personnes (parents, amis, amants...) ou d'exercer une activité (cultuelle, philosophique...). Lorsque le médecin estime par une motivation adaptée que cette rencontre fait courir un risque pour la santé de l'intéressé, il lui est loisible de prononcer une interdiction individuelle. Cette dernière n'est pas une sanction mais une modalité de prise en charge. La légalité de ce refus est d'ailleurs contestable devant le juge, au besoin par le biais de référé. Il est donc possible d'envisager que certains patients se voient refuser à certaines périodes un droit à l'intimité sexuelle, mais uniquement pour raisons médicales. Comme un placement en chambre d'isolement ou l'usage de la contention, l'interdiction de s'isoler est donc temporairement envisageable.

40. Art. 3211-3 CSP : «En tout état de cause, [toute personne faisant l’objet de soins psychiatriques en application des chapitres II et III du présent titre] dispose du droit: $1^{\circ}$ De communiquer avec les autorités mentionnées à l'article L. 3222-4; ${ }^{\circ}$ De saisir la commission prévue à l'article L. $3222-5$ et, lorsqu'elle est hospitalisée, la commission mentionnée à l'article L. 1112-3; $3^{\circ}$ De porter à la connaissance du Contrôleur général des lieux de privation de liberté des faits ou situations susceptibles de relever de sa compétence; $4^{\circ}$ De prendre conseil d'un médecin ou d'un avocat de son choix; $5^{\circ}$ D'émettre ou de recevoir des courriers; $6^{\circ}$ De consulter le règlement intérieur de l'établissement et de recevoir les explications qui s'y rapportent; $7^{\circ}$ D'exercer son droit de vote; $8^{\circ}$ De se livrer aux activités religieuses ou philosophiques de son choix $"$. 


\section{B. Informer la personne dans un langage accessible}

Le patient en psychiatrie n'est pas un usager ordinaire dont on peut attendre qu'il maîtrise correctement la langue française écrite et orale. Son état de santé et parfois son origine sont quelquefois des obstacles à une bonne prise en charge. À plusieurs reprises le législateur impose une obligation d'information particulière à la charge de l'établissement dont le non-respect est susceptible d'engager la responsabilité administrative. L'article L. 3211-2-1 du Code de la santé dispose ainsi que:

Pour l'établissement et la modification du programme de soins, le psychiatre de l'établissement d'accueil recueille l'avis du patient lors d'un entretien au cours duquel il donne au patient l'information prévue à l'article L. 3211-3 et l'avise des dispositions du III du présent article et de celles de l'article L. 3211-11.

Cet article L. 3211-3 du Code de la santé publique impose qu'

Avant chaque décision prononçant le maintien des soins [...] ou définissant la forme de la prise en charge [...] la personne faisant l'objet de soins psychiatriques est, dans la mesure où son état le permet, informée de ce projet de décision et mise à même de faire valoir ses observations, par tout moyen et de manière appropriée à cet état.

Concrètement, l'établissement devra donc apporter la preuve qu'il a tout mis en œuvre pour transmettre l'information, y compris lorsque le patient ne peut consentir et qu'il ne comprend pas le français «juridique et psychiatrique».

Dans une affaire portant sur le décès plusieurs semaines après son hospitalisation d'une personne ne maîtrisant pas le français et entrée à l'hôpital volontairement suite à une dégradation générale de son état de santé, les juges ont reconnu la faute de l'établissement suite au décès d'une patiente ${ }^{41}$. Il s'agissait d'une femme hospitalisée dans un service d'hépato-gastro-entérologie:

[...] les investigations paracliniques se sont révélées négatives tant sur le plan biologique que viscéral; [...] elle a également bénéficié [...] d'une consultation psychiatrique qui a permis de diagnostiquer un syndrome anxio-dépressif réactionnel au décès d'un membre de sa famille.

Avant de quitter l'établissement, «plusieurs médicaments destinés notamment à traiter son état dépressif lui ont été prescrits pour une durée d'un mois et une hospitalisation pour une coloscopie a été programmée».
La patiente s'est donné la mort, alors qu'elle prenait le traitement prescrit. Sa famille considère que l'établissement a commis une faute à l'origine du décès. Les juges bordelais vont reconnaître la responsabilité de l'établissement compte tenu de la gravité du «tableau psychiatrique» de la patiente. Selon eux, deux éléments permettent de retenir la faute et de présumer le lien de causalité entre cette dernière et le passage à l'acte. Tout d'abord, l'établissement ne pouvait ignorer l'état dépressif majeur de la patiente, puisque celui-ci avait été diagnostiqué lors du séjour de la patiente dans l'établissement. De ce point de vue, le service avait donc parfaitement fonctionné. Par contre, lorsqu'un tel diagnostic est réalisé, il n'est pas possible de se contenter de rédiger une simple ordonnance prescrivant un traitement pour un mois.

Pour la cour, le médecin psychiatre aurait dû «prévoir un suivi de la patiente, soit en consultation ambulatoire de psychiatrie, soit dans le secteur libéral». Faute d'avoir prescrit un suivi adapté de la patiente, le centre hospitalier a commis une faute. Ensuite, la situation de la patiente n'a pas bien été prise en compte par l'équipe soignante, ce qui a considérablement réduit les chances de parvenir à une prise en charge efficace. L'information délivrée à la patiente n'était manifestement pas adaptée. Ne sachant pas lire (ce que l'établissement aurait dû vérifier) et s'exprimant dans un français approximatif (ce que l'équipe soignante ne pouvait manifestement ignorer sachant qu'elle est tout de même restée dans le service plus de quinze jours), la patiente n'a pas pu recevoir une information adaptée sur son état, "sur les risques du traitement prescrit et les précautions à prendre». Ce défaut d'information, cumulé à la faute de prescription, suffit à engager la responsabilité de l'établissement.

Cette douloureuse affaire illustre parfaitement les difficultés quotidiennes que rencontrent les services hospitaliers, et pas seulement en psychiatrie, lorsqu'ils doivent accueillir des patients qui s'éloignent du modèle idéal typique de l'usager du service public ayant servi de référence à l'élaboration de la norme. L'information du patient suppose d'avoir préalablement évalué son degré de compréhension du langage écrit et oral, mais aussi sa capacité à conceptualiser et à se projeter dans l'avenir. L'organisation de la présence d' "interprètes » compétents (en droit et en psychiatrie) occupe pour l'instant les différents comités d'éthique. Elle va désormais retenir l'attention des chefs d'établissement et bientôt celle des juges. Il est grand temps que le législateur élabore un texte sur la prise en charge de la maladie mentale et pas uniquement sur l'usage de la contrainte en psychiatrie. 